\title{
En Face Optical Coherence Tomography to Detect and Measure Geographic Atrophy
}

\author{
Elisabetta Pilotto, ${ }^{1}$ Francesca Guidolin,${ }^{1}$ Enrica Convento,${ }^{1}$ Rachele Antonini, ${ }^{1}$ \\ Francesco Giuseppe Stefanon, ${ }^{1}$ Raffaele Parrozzani, ${ }^{2}$ and Edoardo Midena ${ }^{1,2}$
}

${ }^{1}$ Institute of Ophthalmology, University of Padova, Padova, Italy

${ }^{2}$ G.B. Bietti Foundation, IRCCS, Roma, Italy

Correspondence: Edoardo Midena, Institute of Ophthalmology, University of Padova, via Giustiniani 2, 35128 Padova, Italy; edoardo.midena@unipd.it.

Submitted: May 29, 2015

Accepted: November 5, 2015

Citation: Pilotto E, Guidolin F, Convento $\mathrm{E}$, et al. En face optical coherence tomography to detect and measure geographic atrophy. Invest Ophthalmol Vis Sci. 2015;56:81208124. DOI:10.1167/iovs.15-17366
Purpose. To detect and quantify geographic atrophy (GA) secondary to age-related macular degeneration using en face optical coherence tomography (OCT) and to correlate it to GA measured with fundus autofluorescence (FAF).

MethoDs. Twenty-four consecutive patients (27 eyes) were studied with both standard (STD)and enhanced depth imaging (EDI)-OCT. En face OCT images were obtained at the outer retinal layer $(\mathrm{OR})$ and at the choroidal layer $(\mathrm{CH})$ level for both STD- and EDI-OCT. Areas of GA were measured on the en face OCT images and were correlated with the GA areas measured on blue (B)- and near infrared (NIR)-wavelength FAF images.

RESults. The intraoperator agreement in GA measurement was excellent with en face OCT at both $\mathrm{OR}$ and $\mathrm{CH}$ levels (intraclass correlation coefficient [ICC] $=0.99$ in EDI and 0.98 in STD at OR level; 0.99 in EDI and 0.99 in STD at $\mathrm{CH}$ level). The interoperator agreement was excellent at OR level (ICC $=0.97$ in EDI and 0.98 in STD), good at CH level (ICC $=0.95$ in EDI, 0.90 in STD). The geographic atrophy area, at both B-FAF and NIR-FAF, was significantly equivalent to the GA area at OR level (B-FAF versus SDT-OR and EDI-OR: $P=0.0057$ and 0.0090 , respectively; NIR-FAF versus STD-OR and EDI-OR: $P=0.0131$ and 0.0036 , respectively), but not at $\mathrm{CH}$ level.

Conclusions. En face OCT is a reliable method to detect and quantify GA, particularly when analyzed at the OR level, where the photoreceptors' loss creates an abrupt transition in OCT reflectivity.

Keywords: geographic atrophy, age-related macular degeneration, en face optical coherence tomography, fundus autofluorescence, near infrared fundus autofluorescence
$\mathrm{G}$ eographic atrophy (GA) represents the atrophic late-stage J manifestation of dry AMD. ${ }^{1}$ Geographic atrophy is characterized by the loss of photoreceptors, retinal pigment epithelium, and choriocapillaris within the macula. Therefore, GA is a significant cause of moderate to severe central visual loss. The areas of GA slowly progress over time. ${ }^{2}$ Geographic atrophy is usually assessed by color fundus images or, more recently, by fundus autofluorescence (FAF), using both blue and near-infrared FAF (B-FAF and NIR-FAF). ${ }^{3-5}$

Optical coherence tomography (OCT) is a noninvasive tool that provides retinal cross-sectional images of the posterior pole. More recently, the introduction of spectral-domain (SD)OCT has provided further improvement in imaging speed and resolution compared with previous time-domain OCT. The simultaneous recording of confocal scanning laser ophthalmoscopy and SD-OCT images in one instrument, with an exact topographic overlay during image acquisition, allows for accurate orientation of cross-sectional SD-OCT scans at anatomic sites of interest, and serial examinations at the same location over time. ${ }^{6}$ On the SD-OCT B-scan GA is characterized by a choroidal signal enhancement that correlates well with the hypoautofluorescent area measured on FAF, ${ }^{6,7}$ The advent of enhanced-depth imaging SD-OCT (EDI-OCT) allows a better in vivo examination of the choroid than standard SD-OCT. Enhanced-depth imaging OCT has reappraised the clinical interest toward choroid in AMD. ${ }^{9,10}$ A new approach for imaging the fundus uses the en face OCT mode. As a complement to conventional B-scan OCT, en face OCT provides a coronal full macular view at different depth levels, and may provide additional anatomic insight of this disease. ${ }^{11}$ The en face images differ according to the different position of the coronal scan into the retina or in choroid. Therefore, en face OCT may provide additional morphologic insight in many macular diseases. ${ }^{12-14}$ The increased OCT choroidal signal associated with GA results in the visualization of GA areas on choroidal $(\mathrm{CH})$ en face OCT image, whereas loss of OCT signal in the outer retina (OR) also allows visualization of GA on en face OCT images. ${ }^{15}$

The purpose of this study was to detect and quantify GA by means of SD-OCT using both standard (STD) and EDI OCT acquisition mode, viewing en face OCT images at different depth levels and to correlate the en face OCT GA area to the GA area obtained by means of FAF

\section{MeTHODS}

\section{Patients}

Patients affected by GA were consecutively enrolled in this observational, cross-sectional study. Patients were aged older 

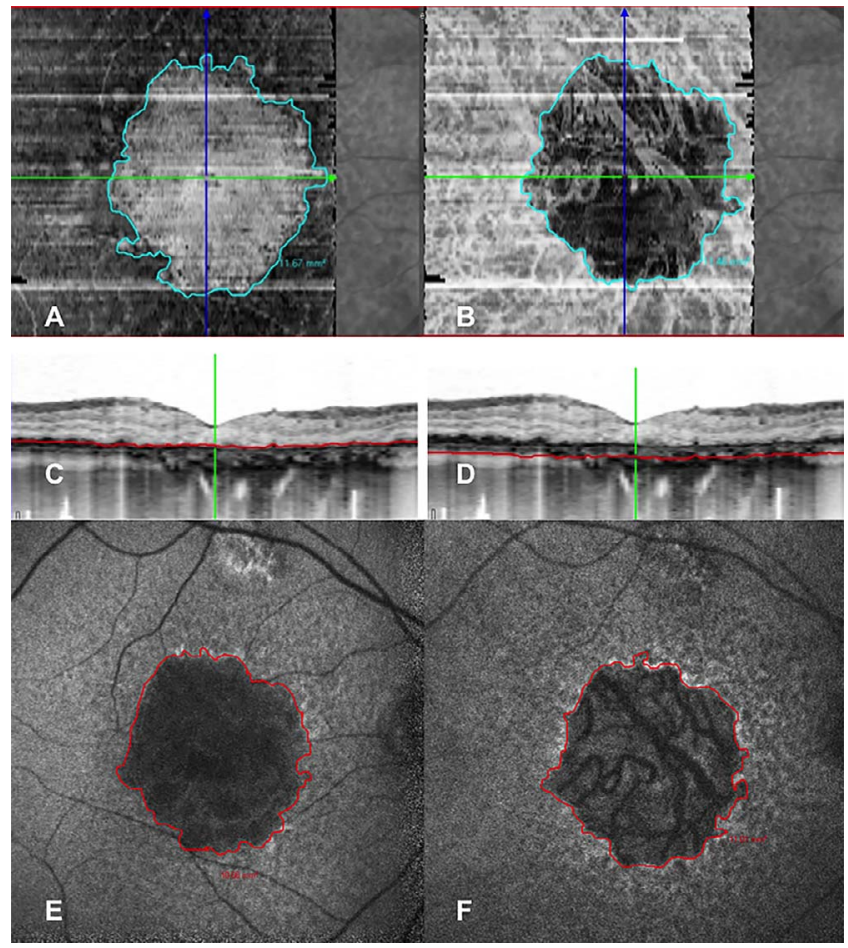

Figure 1. Geographic atrophy measured on the $\mathrm{OR}$ and $\mathrm{CH}$ en face EDI-OCT images (A, B) in an 82-year-old female with geographic atrophy secondary to age-related macular degeneration. The first image (A) is obtained considering a segmentation line (red line) above RPE passing through the outer retinal layers $(\mathbf{C})$; in the second image (B), the segmentation line (red line), parallel to the previous one, is located below RPE in the choroid (D). Geographic atrophy measured on the BFAF and NIR-FAF fundus autofluorescence images $(\mathbf{E}, \mathbf{F})$ in the same patient. Measurement of GA area was $11.67 \mathrm{~mm}^{2}$ on the OR en face OCT image, $11.46 \mathrm{~mm}^{2}$ on the CH en face OCT image, $10.66 \mathrm{~mm}^{2}$ on the B-FAF image, and $11.01 \mathrm{~mm}^{2}$ on the NIR-FAF image.

than 55 years. The inclusion criteria were presence of unifocal or multifocal GA secondary to AMD in at least one eye at fundus examination. Exclusion criteria were significant media opacities, clinical evidence of choroidal neovascularization, any evidence of diabetic retinopathy, myopia $>6$ diopters (D), glaucoma, previous macular laser treatment, signs or history of hereditary retinal dystrophy. Eyes in which macular GA was confluent with the peripapillary atrophy were excluded. Patients were also excluded if GA extended outside the central OCT scanning area (a square centered on the fovea with dimensions $6 \times 6 \mathrm{~mm})$.

Fluorescein angiography was performed only if there were presumed signs of neovascular AMD (extracellular fluid, hemorrhages, exudates, or fibrosis), if confirmed eyes were excluded from the study. This study was conducted in accordance with the tenets of the Declaration of Helsinki, and with the approval of our Institutional Ethical Committee.

After a detailed explanation of the purpose of this study, all enrolled patients signed a written consent form. The ophthalmologic examination consisted of: refraction and best corrected visual acuity determination, anterior segment examination, 90-diopter lens biomicroscopy, fundus photography, FAF, and OCT. All imaging modalities were performed under mydriasis.

\section{Fundus Autofluorescence}

Fundus autofluorescence was performed as previously described. ${ }^{16}$ Briefly, in vivo acquisition of blue (B)- and near- infrared (NIR)-FAF was performed with a confocal scanning laser ophthalmoscope (Heidelberg Retinal Angiograph, HRA2; Heidelberg Engineering $\mathrm{GmbH}$, Heidelberg, Germany). The automatic real-time averaging mode (ART), when activated, allows for adjustment of the recorded frames to obtain averaged scans, which enhances image quality by reducing movement artifacts and optimizes the signal-to-noise ratio. ${ }^{6}$

For the purpose of this study, the ART was set at 100 frames to obtain best quality of FAF (it means that each image was averaged from 100 acquired frames). To measure the areas of GA, unifocal or multifocal circular areas of reduced FAF signal were measured by outlining them using the image analysis software (Heidelberg Eye Explorer; Heidelberg Engineering $\mathrm{GmbH}$ ), which measures region overlay. ${ }^{16}$ Using this tool, pixel area is automatically converted into square millimeters. The total area was calculated for each single image. In order to assess the intraoperator repeatability, the grader repeated the measures twice, at least 24 hours apart.

\section{En Face SD-OCT}

En face OCT was obtained using the imaging system (Spectralis; Heidelberg Engineering $\mathrm{GmbH}$ ). The device uses a superluminescence diode emitting a scan beam at a wavelength of $870 \mathrm{~nm}$. The retina is scanned at a speed of $40,000 \mathrm{~A}$-scans/second with an axial resolution of $3.9 \mu \mathrm{m}$ and a transversal resolution of $14 \mu \mathrm{m}$. The eye tracking and ART of the imaging system (Spectralis; Heidelberg Engineering $\mathrm{GmbH}$ ) were used throughout the study. The eye tracker enables each OCT scan to be registered and locked to a reference image. We set ART at 50 frames.

For the purpose of the study $20 \times 20^{\circ}(5.90 \times 5.90 \mathrm{~mm})$ volume scans centered onto the fovea, using both EDI and STD OCT scan modality, were acquired. Ninety-seven horizontal scans $60 \mu \mathrm{m}$ apart were obtained.

Recently, SD-OCT devices allow to visualize transverse coronal scans (C-scans), also called en face images. En face images differ according to different position of the coronal scan into the retina or in the choroid. Using the automatic retinal segmentation of the device to define the depth level, two different en face OCT images were generated: the outer retinal (OR) and the choroidal $(\mathrm{CH})$ en face images. The en face OR image was obtained considering a segmentation reference line passing through the outer retinal layers, located at $25 \mu \mathrm{m}$ from the Bruch's membrane. The en face $\mathrm{CH}$ image was obtained considering a segmentation line, parallel to the previous one, located in the choroid, at $50 \mu \mathrm{m}$ from the Bruch's membrane (Fig. 1). The automatic segmentation line, at the level of the Bruch's membrane, was used as the reference line. This line was manually reviewed if algorithm failed.

Both outer retinal and $\mathrm{CH}$ en face images were obtained both for STD and for EDI volume scans, therefore four different en face images were visualized and analyzed for every single studied eye.

To measure the GA area, two independent experienced graders (RA, FG) manually outlined the areas of GA on the en face images using the image analysis software (Heidelberg Eye Explorer; Heidelberg Engineering GmbH; Fig. 1). ${ }^{16}$ In order to assess the intraoperator repeatability, one grader (RA) repeated the measures twice, at least 24 hours apart. Both graders were blind from the FAF images, which were measured by a different experienced operator (FGS).

\section{Statistical Evaluation}

Analysis was performed to assess intra- and intergrader agreement of GA measurements using en face imaging 
TABLE 1. Measurement of GA Area in FAF and in En Face OCT

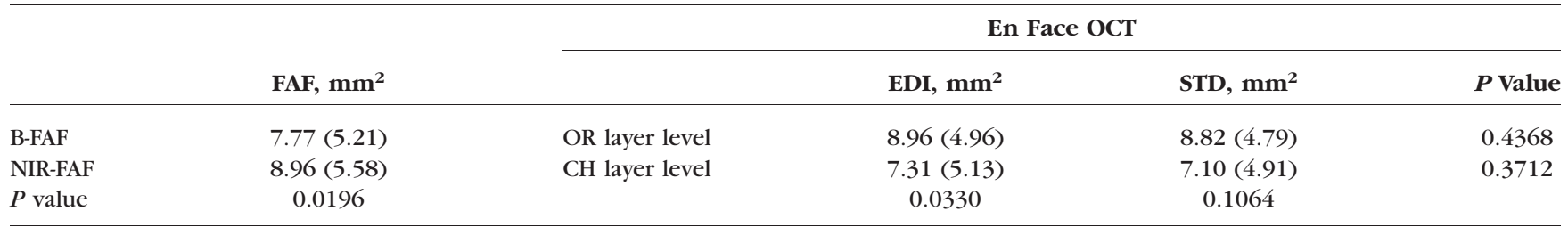

All values are mean (SD) unless otherwise indicated. $P$ value, paired Student's $t$-test significance value.

modalities. In addition to intraclass correlation coefficient (ICC) calculations, Bland-Altman plots were constructed to determine limits of agreement.

Statistical analysis to assess the correlation between FAF and en face OCT was done using the intraclass correlation coefficient calculations, Bland-Altman plots and the equivalence test. We evaluated the equivalence of STD and EDI, with both $\mathrm{OR}$ and $\mathrm{CH}$ en face measures, versus NIR-FAF and B-FAF by means of two one-sided test method. The null hypothesis (HO) was the means of the two samples differ. The alternative hypotheses (H1) was the means of the two samples were equivalent. Our aim was to prove the alternative hypotheses (H1).

A range of $\pm 15 \%$ of FAF mean values was assumed as interval of clinical equivalence. The mean value and $95 \%$ confidence interval of the differences between EDI/STD and FAF were calculated. We accepted the equivalence assessment if the $95 \%$ confidence interval of the difference between the mean values of EDI or STD and FAF resulted completely within the equivalence interval.

The data obtained were analyzed using PROC MIXED procedure with statistical software (SAS 9.2; SAS Institute, Cary, NC, USA). A value of $P<0.05$ was considered statistically significant.

\section{Results}

Twenty-four consecutive patients (27 eyes) affected by GA were studied. Mean age was $80.38 \pm 6.47$ (range, 69-98). Mean GA area was $7.77 \pm 5.21 \mathrm{~mm}^{2}$ measured on B-FAF and $8.96 \pm 5.58 \mathrm{~mm}^{2}$ on NIR-FAF $(P=0.0196)$. On en face image, using STD OCT acquisition mode, mean GA area was $8.82 \pm$ $4.79 \mathrm{~mm}^{2}$ at OR layer and $7.10 \pm 4.91 \mathrm{~mm}^{2}$ at $\mathrm{CH}$ layer level $(P$ $=0.1064$ ). Using EDI OCT acquisition mode, mean GA area was $8.96 \pm 4.96 \mathrm{~mm}^{2}$ at $\mathrm{OR}$ level and $7.31 \pm 5.13 \mathrm{~mm}^{2}$ at $\mathrm{CH}$ level $(P=0.0330$; Table 1).

\section{Intraoperator Repeatability}

The intraoperator repeatability was excellent both on NIR-FAF and on B-FAF modalities (ICC $=0.99$ [95\% CI 0.98-0.99] and ICC $=0.99$ [95\% CI 0.998-0.999], respectively).

On en face OCT, using STD acquisition mode, intraoperator repeatability was excellent both at $\mathrm{OR}$ and at $\mathrm{CH}$ level $(\mathrm{ICC}=$

TABLE 2. Intraclass Correlation Coefficient Between FAF and En Face OCT

\begin{tabular}{lcc}
\hline & B-FAF, 95\% (CI) & NIR-FAF, 95\% (CI) \\
\hline OR-STD & $0.95(0.90-0.98)$ & $0.91(0.81-0.96)$ \\
OR-EDI & $0.96(0.91-0.98)$ & $0.93(0.84-0.96)$ \\
CH-STD & $0.89(0.77-0.95)$ & $0.79(0.56-0.90)$ \\
CH-EDI & $0.91(0.81-0.95)$ & $0.82(0.60-0.92)$ \\
\hline
\end{tabular}

ICC, $95 \%$ confidence interval.
0.98 [95\% CI 0.97-0.99] and ICC $=0.99$ [95\% CI 0.98-0.99], respectively).

Using EDI acquisition mode, the intraoperator repeatability was also excellent, both at OR and at $\mathrm{CH}$ level; ICC $0.99(95 \%$ CI 0.991-0.98) and ICC 0.99 (95\% CI 0.98-0.99), respectively.

The intraoperator agreement was corroborated by BlandAltman plots (Supplementary Fig. S1).

\section{Interoperator Agreement}

Using STD OCT acquisition mode, the interoperator agreement was excellent both at $\mathrm{OR}$ level and at $\mathrm{CH}$ level: ICC was 0.98 (95\% CI $0.95-0.99$ ) and 0.90 (95\% CI 0.78-0.95), respectively.

Using EDI OCT acquisition mode, the interoperator agreement was excellent both at $\mathrm{OR}$ and at $\mathrm{CH}$ level; ICC was 0.97 (95\% CI 0.94-0.99] and 0.95 (95\% CI 0.88-0.98), respectively. Bland-Altman plots showed a trend with nonuniformity of variance, as seen in STD modality. The interoperator agreement was corroborated by Bland-Altman plots (Supplementary Fig. S2).

\section{Correlation Between FAF and En Face SD-OCT Images}

Mean GA area measured on B-FAF better correlated to GA area measured at OR level than at $\mathrm{CH}$ level, both on STD and EDI modality. Intraclass correlation coefficient was 0.95 (95\% CI 0.90-0.98) at OR level and 0.89 (95\% CI 0.77-0.95) at CH level on STD modality. Intraclass correlation coefficient was 0.96 (95\% CI 0.91-0.98) at OR level and 0.91 (95\% CI 0.81-0.95) at $\mathrm{CH}$ level on EDI modality (Table 2).

Area of GA measured on NIR-FAF highly correlated to GA area measured at OR level, both on STD and EDI modality (ICC: 0.91 [95\% CI $0.81-0.96$ ] and 0.93 [95\% CI $0.84-0.96$ ], respectively). Moreover, the correlation was good with GA area measured at $\mathrm{CH}$ level, both on STD and on EDI (ICC $=0.79$ [95\% CI 0.56-0.90] and 0.82 [95\% CI 0.60-0.92] respectively; Table 2).

The equivalence test was statistically significant between both FAF images and en face OCT at OR level for both STD and EDI OCT acquisition mode, with the confidence interval being completely inside equivalence interval (B-FAF versus OR-STD, $P$ $=0.0057$; B-FAF versus OR-EDI, $P=0.0090$; NIR-FAF versus $\mathrm{OR}$ STD, $P=0.0131$; NIR-FAF versus OR-EDI, $P=0.0036$; Fig. 2 ).

Moreover, the equivalence test was inconclusive between both FAF images and en face OCT at $\mathrm{CH}$ level (B-FAF versus $\mathrm{CH}-$ STD, $P=0.1203$; B-FAF versus CH-EDI, $P=0.1080$; NIR-FAF versus $\mathrm{CH}-\mathrm{STD}, P=0.5332$; NIR-FAF versus $\mathrm{CH}$-EDI, $P=$ 0.5660; Fig. 2).

\section{Discussion}

As a complement to conventional B-scan OCT, en face OCT is a new imaging modality, which allows a full extent, 2D view of the posterior pole, a topographical analysis and a close comparison with other 2D imaging modalities. In this study, 


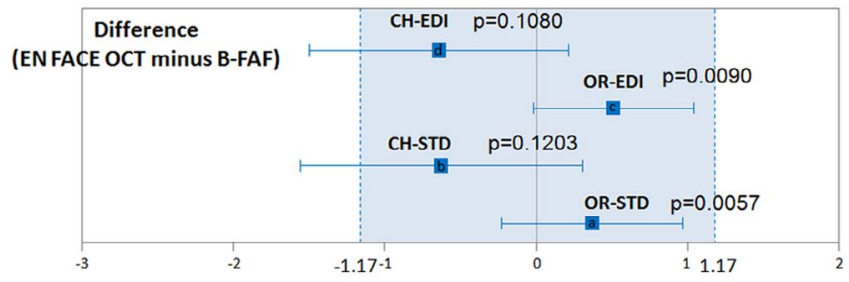

B

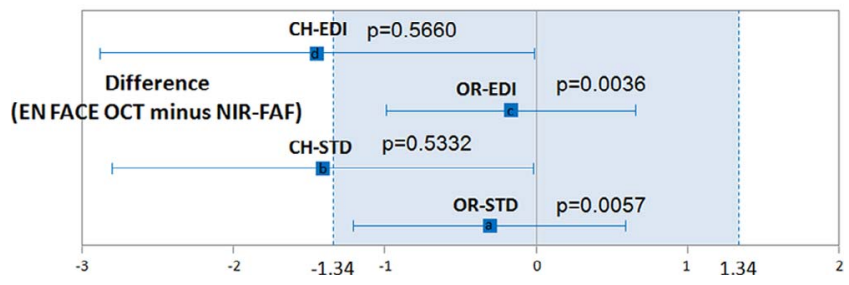

Figure 2. Equivalence tests between B-FAF and NIR-FAF fundus autofluorescence images and en face $\mathrm{OR}$ and $\mathrm{CH}$ en face EDI or STDOCT images. (A) Choroidal EDI: mean difference -0.642 (95\% confidence limits -1.499 to 0.215$), P=0.1080$. Outer retinal EDI: 0.514 ( -0.018 to 1.046$), P=0.0090$. Choroidal STD: $-0.626(-1.560$ to $0.308), P=0.1203$. Outer retinal STD: $0.374(-0.225$ to 0.973$), P=$ 0.0057. (B) Choroidal EDI: $-1.436(-2.621$ to -0.252$), P=0.5660$. Outer retinal EDI: -0.166 ( -0.988 to 0.657$), P=0.0036$. Choroidal STD: -1.393 ( -2.691 to -0.095$), P=0.5332$. Outer retinal STD: -0.306 $(-1.206$ to 0.595$), P=0.0131$.

we used en face OCT to analyze patients with GA secondary to AMD. Using this imaging modality, we found that GA area may be, not only easily visualized, but also quantified, independently to the OCT scan modality (STD or EDI). En face imaging, using a different OCT device (Cirrus HD-OCT; Carl Zeiss Meditec, Inc., Jena, Germany), has been previously used to detect GA area. ${ }^{17}$ Some authors detected GA progression using OCT fundus image. Fundus OCT image is obtained summing the signal of each A-scan and viewing their relative values en face. ${ }^{18}$ Using this approach, GA appears as a bright area, due to the high penetration of light into the choroid where RPE is absent. ${ }^{18}$ The same authors analyzed GA using a different en face image approach, called subretinal (sub)-RPE slab, obtained with the same device. The sub-RPE slab is formed by axially projecting the OCT image data from the region below the RPE fit. ${ }^{19}$ Areas of GA using these two different en face imaging modalities, the OCT fundus image and the sub-RPE slab, are highly correlated. ${ }^{20}$ In the present study, we analyzed en face images obtained with a different SD-OCT device. The area of GA measured with en face OCT was compared with that on BFAF and NIR-FAF images. Our findings show a good correlation between GA area of en face OCT images at OR level, both on BFAF and on NIR-FAF images. Instead, the equivalent test was inconsistent between FAF images and en face OCT at $\mathrm{CH}$ level. Moreover, en face OCT at the OR retinal level allows us to identify reticular pseudodrusen. ${ }^{15}$ Reticular pseudodrusen have been recognized as relevant risk factors in progressing GA, but their recognition was beyond the scope of our study. ${ }^{19,21,22}$

A comparison between en face OCT and B-FAF, which images lipofuscin in the RPE, in the detection of GA has been previously done..$^{17,20,23-25}$ But, to the best of our knowledge, a correlation between en face OCT and NIR-FAF, which visualizes melanin in the RPE and choroid, has never been performed. ${ }^{26,27}$ As previously reported, GA area was larger on NIR-FAF than on B-FAF. ${ }^{4,5,16}$ This finding has been reported in Stargardt disease too. ${ }^{27}$ Interestingly, in the present study, on en face image, mean GA area was larger at OR layer level than at $\mathrm{CH}$ layer level, especially in EDI OCT acquisition mode, which allows a better penetration into both layers. The sequence of events in GA has been variously postulated. ${ }^{28-33}$ Our findings seem to confirm the hypothesis that in GA, photoreceptor atrophy and RPE changes, visualized by en face OCT image at OR level, occur before choriocapillaris loss. ${ }^{34,35}$

The small sample size is a major limitation of this study. But, the strength of our study is that the instrument, avoiding errors due to fully manual OCT segmentation, automatically generated the analyzed en face OCT images.

In conclusion, this study shows that GA can be reproducibly identified and quantified by means of en face OCT imaging. The GA area detected on the en face OCT is comparable not only to that quantified on B-FAF, but also to that quantified on NIR-FAF. The measured GA area is greater at the OR level than at the $\mathrm{CH}$ level, suggesting an earlier degenerative involvement of the outer retina and RPE than the underlying, longer surviving choriocapillaris.

\section{Acknowledgments}

The research contribution by the G.B. Bietti Foundation was supported by Fondazione Roma and Ministry of Health.

Disclosure: E. Pilotto, None; F. Guidolin, None; E. Convento, None; R. Antonini, None; F.G. Stefanon, None; R. Parrozzani, None; E. Midena, None

\section{References}

1. Klein R, Klein BE, Knudtson MD, Meuer SM, Swift M, Gangnon RE. Fifteen-year cumulative incidence of age-related macular degeneration: the Beaver Dam Eye Study. Ophthalmology. 2007; 114:253-262.

2. Schatz H, McDonald HR. Atrophic macular degeneration. Rate of spread of geographic atrophy and visual loss. Ophthalmology. 1989;96:1541-1551.

3. Holz FG, Bindewald-Wittich A, Fleckenstein M, et al.; FAMStudy Group. Progression of geographic atrophy and impact of fundus autofluorescence patterns in age-related macular degeneration. Am J Ophthalmol. 2007;143:463-472.

4. Pilotto E, Guidolin F, Convento E, et al. Fundus autofluorescence and microperimetry in progressing geographic atrophy secondary to age-related macular degeneration. $\mathrm{Br} \mathrm{J} \mathrm{Ophthal-}$ mol. 2013;97:622-626.

5. Kellner U, Kellner S, Weinitz S. Fundus autofluorescence (488 $\mathrm{nm})$ and near-infrared autofluorescence $(787 \mathrm{~nm})$ visualize different retinal pigment epithelium alterations in patients with age-related macular degeneration. Retina. 2010;30:6-15.

6. Sayegh RG, Simader C, Scheschy U, et al. A systematic comparison of spectral-domain optical coherence tomography and fundus auto-fluorescence in patients with geographic atrophy. Ophthalmology. 2011;118:1844-1851.

7. Schmitz-Valckenberg S, Fleckenstein M, Göbel AP, Hohman TC, Holz FG. Optical coherence tomography and autofluorescence findings in areas with geographic atrophy due to agerelated macular degeneration. Invest Ophthalmol Vis Sci. 2011;52:1-6.

8. Spaide R, Koizumi H, Pozzoni M. Enhanced depth imaging spectral-domain optical coherence tomography. $A m \mathrm{~J} O \mathrm{Ob}$ thalmol. 2008;146:496-500.

9. Sim DA, Keane PA, Mehta $\mathrm{H}$, et al. Repeatability and reproducibility of choroidal vessel layer measurements in diabetic retinopathy using enhanced depth optical coherence tomography. Invest Ophthalmol Vis Sci. 2013;54:2893-901.

10. Pilotto E, Guidolin F, Convento E, Stefanon FG, Parrozzani R, Midena E. Progressing geographic atrophy: choroidal thick- 
ness and retinal sensitivity identify two clinical phenotypes. $\mathrm{Br}$ J Opbthalmol. 2015;99:1082-1086.

11. Flores-Moreno I, Arias-Barquet L, Rubio-Caso MJ, Ruiz-Moreno JM, Duker JS, Caminal JM. En face swept-source optical coherence tomography in neovacular age-related macular degeneration. Br J Ophthalmol. 2015;99:1260-1267.

12. Wolff B, Basdekidou C, Vasseur V, Sahel JA, Gaudric A, MaugetFaysse M. "En face" optical coherence tomography imaging in type 2 idiopathic macular teleangiectasia. Retina. 2014;34: 2072-2078.

13. Puche N, Querques G, Balco-Garavito R, et al. En face enhanced depth imaging optical coherence tomography features in adult onset foveomacular vitelliform dystrophy. Graefes Arch Clin Exp Ophthalmol. 2014;252:555-562.

14. Lehmann M, Wolff B, Vasseur V, et al. Retinal and choroidal changes observed with "en face" enhanced-depth imaging OCT in central serous chorioretinopathy. $\mathrm{Br} J$ Ophthalmol. 2013;97:1181-1186.

15. Portella Nunes R, Gregori G, Yehoshua Z, et al. Predicting the progression of geographic atrophy in age-related macular degeneration with SD-OCT en face imaging of the outer retina. Ophthalmic Surg Lasers Imaging Retina. 2013;44:344-359.

16. Pilotto E, Vujosevic S, Melis R, et al. Short wavelength fundus autofluorescence versus near-infrared fundus autofluorescence, with microperimetric correspondence, in patients with geographic atrophy due to age-related macular degeneration. Br J Ophthalmol. 2011;95:1140-1144.

17. Yehoshua Z, Rosenfeld PJ, Gregori G, et al. Progression of geographic atrophy in age-related macular degeneration imaged with spectral domain optical coherence tomography. Ophthalmology. 2011;118:679-686.

18. Lujan BJ, Rosenfeld PJ, Gregori G, et al. Spectral domain optical coherence tomographic imaging of geographic atrophy. Opbthalmic Surg Lasers Imaging. 2009;40:96-101.

19. Stetson PF, Yehoshua Z, Garcia Filho CA. Portella Nunes R, Gregori G, Rosenfeld PJ. OCT minimum intensity as a predictor of geographic atrophy enlargement. Invest Ophthalmol Vis Sci. 2014;55:792-800.

20. Yehoshua Z, Garcia Filho CA, Penha FM, et al. Comparison of geographic atrophy measurements from the OCT fundus image and the sub-RPE slab image. Ophthalmic Surg Lasers Imaging Retina. 2013;44:127-132.

21. Xu L, Blonska AM, Pumariega NM, et al. Reticular macular disease is associated with multilobular geographic atrophy in age-related macular degeneration. Retina. 2013;33:18501862.

22. Marsiglia M, Boddu S, Bearelly S, et al. Association between geographic atrophy progression and reticular pseudodrusen in eyes with dry age-related macular degeneration. Invest Ophthalmol Vis Sci. 2013;54:7362-7369.

23. Delori FC, Dorey CK, Staurenghi G, et al. In vivo fluorescence of the ocular fundus exhibits retinal pigment epithelium lipofuscin characteristic. Invest Opbthalmol Vis Sci. 1995;36: 718-729.

24. Lois N, Owens SL, Coco R, et al. Fundus autofluorescence in patients with age-related macular degeneration and high risk of visual loss. Am J Ophthalmol. 2002;133:341-349.

25. Schmitz-Valckenberg S, Fleckenstein M, Scholl HPN, et al. Fundus autofluorescence and progression of age-related macular degeneration. Surv Opbthalmol. 2009;54:96-117.

26. Gibbs D, Cideciyan AV, Jacobson SG, et al. Retinal pigment epithelium defects in humans and mice with mutation in MY07A: imaging melanosome-specific autofluorescence. Invest Ophthalmol Vis Sci. 2009;50:4386-4393.

27. Greenstein VC, Schuman AD, Lee W, et al. Near-infrared autofluorescence: its relationship to short-wavelength autofluorescence and optical coherence tomography in recessive Stargardt disease. Invest Ophthalmol Vis Sci. 2015;56:32263234 .

28. McLeod DS, Grebe R, Bhutto I, et al. Relationship between RPE and choriocapillaris in age-related macular degeneration. Invest Ophthalmol Vis Sci. 2009;50:4982-4991.

29. Leonard DS, Zhang XG, Panozzo G, et al. Clinicopathologic correlation of localized retinal pigment epithelium debridement. Invest Ophthalmol Vis Sci. 1997;38:1094-1109.

30. Korte GE, Reppucci V, Henkind P. RPE destruction causes choriocapillary atrophy. Invest Ophthalmol Vis Sci. 1984;25: 1135-1145.

31. Curcio CA, Medeiros NE, Millican CL. Photoreceptor loss in age-related macular degeneration. Invest Ophthalmol Vis Sci. 1996;37:1236-1249.

32. Bearelly S, Chau FY, Koreishi A, et al. Spectral domain optical coherence tomography imaging of geographic atrophy margins. Ophthalmology. 2009;116:1762-1769.

33. Whitmore SS, Sohn EH, Chirco KR, et al. Complement activation and choriocapillaris loss in early AMD: implications for pathophysiology and therapy. Prog Retin Eye Res. 2015;45: 1-29.

34. Curcio CA, Saunders PL, Younger PW, Malek G. Peripapillary chorioretinal atrophy: Bruch's membrane changes and photoreceptor loss. Ophthalmology. 2000;107:334-343.

35. Biesemeier A, Taubitz T, Julien S, Yoeruek E, Schraermeyer U. Choriocapillaris breakdown precedes retinal degeneration in age-related macular degeneration. Neurobiol Aging. 2014;35: 2562-2573. 